\title{
Radial-probe EBUS for the diagnosis of peripheral pulmonary lesions
}

\author{
Juliana Guarize1, Stefano Donghi', Maurício Guidi Saueressig ${ }^{1,2,3}$
}

We would like to make some comments regarding the article by Jacomelli et al., (1) which describes an initial experience using radial-probe endobronchial ultrasound (EBUS) for the investigation of 51 pulmonary lesions.

First, we have found data inconsistencies in Tables 1 and 2 in the aforementioned article. The authors reported, both in the body of the text and in Table 1 , that the diagnostic sensitivity of radial-probe EBUS for radial-probe EBUS-visible nodules was $74.1 \%$ : 20 surgically confirmed diagnoses among 27 radial-probe EBUS-visible nodules. However, in the second column of Table 2, we found that 21 diagnosed cases of radial-probe EBUS-visible nodules (10 cases of malignant disease and 11 cases of nonmalignant disease) were listed, which differs from the sum of 20 cases recorded in the last row of the same column. Also in Table 2, one case of hamartoma was erroneously included among the malignant nodules.

The study reports, in its results, the value of $66.7 \%$ (34 diagnoses in 51 cases) as the overall sensitivity (diagnostic yield) of radial-probe EBUS for malignant and benign diseases. However, we do not understand why, for the calculation of this sensitivity, 3 radial-probe EBUS-invisible lesions were included with the 31 radial-probe EBUS-visible lesions that were diagnosed by this method.

In addition, the prevalence of neoplasia, a relevant factor for the analysis of the diagnostic yield, ${ }^{(2)}$ was not informed; nor was the final diagnosis of the 12 radial-probe EBUS-invisible pulmonary lesions. Therefore, the presentation of results by Jacomelli et al.(1) differs in some aspects from that of important publications on the subject. ${ }^{(2,3)}$
In the Department of Interventional Pulmonology of the Instituto Europeo di Oncologia in Milan, Italy, we have used radial-probe EBUS to investigate pulmonary nodules and masses since 2012. We use a miniprobe within a guide sheath (K-203 Guide Sheath Kit; Olympus Medical Systems Corp., Tokyo, Japan) and fluoroscopy for localization and subsequent transbronchial biopsy of the lesions. In all procedures, a pathologist is present in the endoscopy room for rapid on-site cytological evaluation, as previously described. ${ }^{(4)}$ We believe that this is essential for increasing the diagnostic yield of the procedure, as we will describe below.

In 2015, we investigated 161 pulmonary lesions (nodules and masses) using radial-probe EBUS. Three patients who were lost to follow-up were excluded from the statistical analysis. The examination was not diagnostic (its results were nonspecific and unrelated to the final diagnosis, or the bronchial epithelium or lesions were not visible by radial-probe EBUS) in 33 cases (23 cases of malignant disease and 10 cases of benign disease). Among those cases, there were 11 radial-probe EBUS-invisible lesions, which exclusively comprised opacities less than $40 \mathrm{~mm}$. The overall sensitivity of the radial-probe EBUS-guided biopsies was $79 \%$ (108 malignant and 17 benign biopsies). The prevalence of malignant disease was $83 \%$. The sensitivity, specificity, negative predictive value, and accuracy for malignancy among the lesions detected by radial-probe EBUS were, respectively, $88 \%, 100 \%$, $57 \%$, and $89.5 \%$.

Finally, we must emphasize the importance of the article by Jacomelli et al., (1) because, in addition to being the first one on radial-probe EBUS in Brazil, it is an example of use of the growing arsenal of endoscopic tools for the investigation and treatment of pulmonary lesions.

\section{REFERENCES}

1. Jacomelli M, Demarzo SE, Cardoso PF, Palomino AL, Figueiredo VR Radial-probe EBUS for the diagnosis of peripheral pulmonary lesions. Jornal Brasileiro de Pneumologia. 2016;42(4):248-53. http://dx.doi. org/10.1590/S1806-37562015000000079

2. Steinfort DP, Khor $\mathrm{YH}$, Manser RL, Irving LB. Radial probe endobronchial ultrasound for the diagnosis of peripheral lung cancer: systematic review and meta-analysis. Eur Respir J. 2011;37(4):902-10. http://dx.doi. org/10.1183/09031936.00075310
3. Eberhardt R, Ernst A, Herth FJ. Ultrasound-guided transbronchial biopsy of solitary pulmonary nodules less than $20 \mathrm{~mm}$. Eur Respir J. 2009;34(6):1284-7. http://dx.doi.org/10.1183/09031936.00166708

4. Guarize J, Pardolesi A, Donghi S, Filippi N, Casadio C, Midolo V, et al. Endobronchial ultrasound for mediastinal staging in lung cancer patients. Multimed Man Cardiothorac Surg. 2014;2014. pii: mmu021. http:// dx.doi.org/10.1093/mmcts/mmu021

1. Divisione di Chirurgia Toracica, Istituto Europeo di Oncologia, Milano, Italia. 


\section{AUTHORS' REPLY}

\section{Marcia Jacomelli', Sergio Eduardo Demarzo', Paulo Francisco Guerreiro Cardoso², Addy Lidvina Mejia Palomino ${ }^{1}$, Viviane Rossi Figueiredo ${ }^{1}$}

We are grateful for the criticisms of our study that was published in the JBP in 2016. (1) We have reviewed all cases and interpretations and requested the necessary corrections to improve the description of the results.

Of all 54 patients who underwent bronchoscopy with radial-probe endobronchial ultrasound (EBUS) for the diagnosis of pulmonary lesions, 3 were excluded because they were lost to follow-up and we could not perform comparisons with the final results obtained by other methods or by clinical follow-up. Therefore, there remained 51 patients who were included in the analysis (Table 1 ). Among those 51 cases, we made 34 diagnoses by the bronchoscopic procedure, all of which were confirmed by other methods or by clinical-radiological follow-up, amounting to an overall diagnostic yield of $66.7 \%$ (nodules and masses). We divided those 51 cases into radial-probe EBUS-visible lesions ( $n=39$ ) and radial-probe EBUS-invisible lesions ( $n=12)$. Among the radial-probe EBUS-visible lesions, we made a total of 31 diagnoses (79.5\%), including 20 nodules (74.1\%) and 11 masses (91.7\%). Among the 12 radial-probe EBUS-invisible lesions, we made only 3 diagnoses (25\%). This shows that, if the lesion is visible by radial-probe EBUS, there is greater likelihood of making a final diagnosis by the bronchoscopic methods. ${ }^{(2,3)}$ A correction must be made to the last row of Table 1, which should read: not identified by radial-probe EBUS.

In Table 2, hamartoma was erroneously placed among the cases of malignant disease, which were originally designated "tumors" and therefore included all benign and malignant cases. Also in Table 2, in the row that reads inflammatory disease, we made a total of 2 diagnoses by the bronchoscopic method that were confirmed ( $n=2 ; 66.7 \%)$, meaning that the total number of diagnoses made in the pulmonary nodule group amounts to 20 diagnoses. These errors must be corrected in Table 2 .

Regarding rapid on-site evaluation of the specimen by a pathologist and fluoroscopy, we know how important these techniques are to the procedure; however, they are not available in the majority of our procedures. In addition, guide sheaths are not yet available for use in Brazil, which largely precludes the collection of adequate material in some cases.

\section{REFERENCES}

1. Jacomelli M, Demarzo SE, Cardoso PF, Palomino AL, Figueiredo VR. Radial-probe EBUS for the diagnosis of peripheral pulmonary lesions Jornal Brasileiro de Pneumologia. 2016;42(4):248-53. http://dx.doi. org/10.1590/S1806-37562015000000079

2. Huang CT, Ho CC, Tsai YJ, Yu CJ, Yang PC. Factors influencing visibility and diagnostic yield of transbronchial biopsy using endobronchial ultrasound in peripheral pulmonary lesions.

Respirology. 2009;14(6):859-64. http://dx.doi.org/10.1111/j.1440 1843.2009.01585.x

3. Steinfort DP, Khor $\mathrm{YH}$, Manser RL, Irving LB. Radial probe endobronchial ultrasound for the diagnosis of peripheral lung cancer: systematic review and meta-analysis. Eur Respir J. 2011;37(4):90210. http://dx.doi.org/10.1183/09031936.00075310

1. Serviço de Endoscopia Respiratória, Divisão de Pneumologia, Instituto do Coração, Hospital das Clínicas, Faculdade de Medicina, Universidade de São Paulo, São Paulo (SP) Brasil.

2. Departamento de Cardiopneumologia, Disciplina de Cirurgia Torácica, Instituto do Coração, Hospital das Clínicas, Faculdade de Medicina, Universidade de São Paulo, São Paulo (SP) Brasil. 